\title{
Radio communication via Near Vertical Incidence Skywave propagation: an overview
}

\author{
Ben A. Witvliet ${ }^{1,2}$ - Rosa Ma Alsina-Pagès ${ }^{3}$
}

Published online: 21 February 2017

(C) The Author(s) 2017. This article is published with open access at Springerlink.com

\begin{abstract}
Near Vertical Incidence Skywave (NVIS) propagation can be used for radio communication in a large area (200 km radius) without any intermediate man-made infrastructure. It is therefore especially suited for disaster relief communication, communication in developing regions and applications where independence of local infrastructure is desired, such as military applications. NVIS communication uses frequencies between approximately 3 and $10 \mathrm{MHz}$. A comprehensive overview of NVIS research is given, covering propagation, antennas, diversity, modulation and coding. Both the bigger picture and the important details are given, as well as the relation between them.
\end{abstract}

Keywords Radio communication - Emergency communications · Radio wave propagation · Ionosphere · NVIS · Antennas $\cdot$ Diversity $\cdot$ Modulation

Ben A. Witvliet

ben.witvliet@agentschaptelecom.nl;

B.A.Witvliet@utwente.nl

Rosa Ma Alsina-Pagès

ralsina@salleURL.edu

1 Spectrum Management Department, Radiocommunications Agency Netherlands, P. O. Box 450, 9700 AL Groningen, The Netherlands

2 Telecommunication Engineering Group, University of Twente, P. O. Box 217, 7500 AE Enschede, The Netherlands

3 GTM - Grup de Recerca en Tecnologies Mèdia, La Salle Universitat Ramon Llull, c/Quatre Camins, 30, 08022 Barcelona, Spain

\section{Introduction}

Recently, interest in radio communication via Near Vertical Incidence Skywave (NVIS) propagation has revived, not in the least because of its role in emergency communications in large natural disasters that took place in the last decade [1-3]. The NVIS propagation mechanism enables communication in a large area without the need of a network infrastructure, satellites or repeaters. This independence of local infrastructure is essential for disaster relief communications, when the infrastructure is destroyed by a large scale natural disaster, or in remote regions where this infrastructure is lacking. In military communications, where independence of local infrastructure is equally important, communications via NVIS propagation have always remained important next to troposcatter and satellite links.

For NVIS propagation, electromagnetic waves are sent nearly vertically towards the ionosphere, the ionized upper part of the Earth's atmosphere. With appropriate frequency selection, these waves are reflected back to Earth [4], as shown in Fig. 1. The great reflection height of $80-350 \mathrm{~km}$ results in a large footprint and homogeneous field strength across that footprint. Due to the steep radiation angles large objects such as mountain slopes or high buildings cannot block the radio path [5]. Typical frequencies are between 3 and $10 \mathrm{MHz}$. The term 'Near Vertical Incidence Skywave' was first mentioned by Rufenach et al. [6], although others claim that Perlman [7] named the propagation mechanism. The latter used the term 'Nearly Vertical Incidence Skywave'.

NVIS propagation may be used to cover an area with a $200 \mathrm{~km}$ radius using low power and simple antennas [4]. As can be derived from the measurements in [8], a modest transmit power of $20 \mathrm{~W}$ in a dipole antenna will produce more than $30 \mathrm{~dB}$ signal-to-noise ratio (SNR) in a $3 \mathrm{kHz}$ bandwidth, which is sufficient for data transfer speeds of at least 


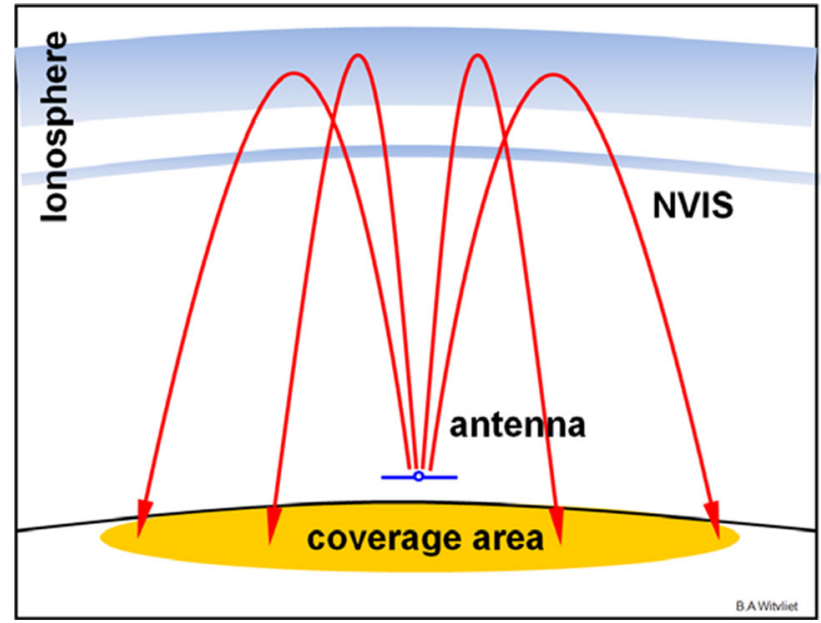

Fig. 1 In Near Vertical Incidence Skywave (NVIS) communications, electromagnetic waves sent nearly vertically towards the ionosphere are reflected and land in the area the transmitter

$8 \mathrm{kbps}$, possibly even $16 \mathrm{kbps}$ [9]. This SNR is constant over the entire coverage area and does not decay with increasing distance.

\subsection{Historical perspective}

The first documented scientific research into NVIS propagation was performed by Appleton [10] to prove the existence of the 'Heavyside layer' by fringe measurements over $100 \mathrm{~km}$. In another experiment [11] he analyzes the difference in time delay between pulses transmitted over a distance of $5 \mathrm{~km}$, arriving both via groundwave and skywave. These experiments were performed to verify theories on radio wave propagation in the ionosphere. Since then, vertical sounding has been used extensively for research into properties of the ionosphere, with increasing precision and sophistication.

Most ionospheric radio propagation research between 1930 and 1950 aimed at improving long distance telecommunications using 'short waves' (wavelength $<100 \mathrm{~m}$ ). Ionospheric radio communication proved very effective, and a world-wide public radiotelephony network was formed [12]. These HF radio links were gradually phased out when satellite transponders [13] and transatlantic cables with sufficient bandwidth for telephony [14] became available from 1956 onward.

NVIS (short distance) propagation was rediscovered in World War II as an essential means to establish communications in large war zones such as the D-Day invasion in Normandy [4, pp. 122-124] [15], and a substantial volume of army sponsored research on NVIS field communications has been published since, especially between 1966 and 1973 $[16,17]$.

Modern radio and signal processing hardware enable new modulation and coding solutions, and automatic link estab- lishmen techniques. The use of HF Multiple Input Multiple Output (MIMO) to increase channel capacity was first proposed by [18]. Research into the improvement of landmobile and airmobile NVIS antennas is also from the last two decades $[19,20]$.

\subsection{NVIS applications}

In the aftermath of a large scale natural disaster, often all telecommunication networks are disabled, the electrical supply is disrupted and the roads are blocked with debris or flooded $[21,22]$. As our society is dependent on communication, the total disruption of communication in the aftermath of a major disaster has a devastating impact on society [23,24]. NVIS communications have proven an excellent alternative for first responders in several recent large natural disasters, such as the Indian Ocean Tsunami of 2004 and the flooding of New Orleans after Hurricane Katrina in 2005 [25].

In humanitarian projects, NVIS propagation can provide low cost communications in poor and remote regions. A lot of progress has been made in connecting the developing world [26,27], but areas remain where the telecommunication infrastructure is nonexistent, unreliable or inaccessible due to lack of financial means. In such regions voice and data traffic for tele-medicine and tele-education is often realized using NVIS propagation [28]. NVIS communications have always remained important as one of the alternatives in military communications. However, recent technological improvements have increased interest. Modulation systems are developed that are optimized for the ionospheric channel to increase data throughput, and modern Automatic Link Establishment (ALE) protocols [29] enable integration of NVIS links in heterogeneous communications networks.

While coverage in scientific media is scarce, medium wave and shortwave broadcasting in the tropical zone exploits NVIS propagation to cover the area around the transmitter in a power efficient way [30]. Sea-going sailing yachts often also employ digital modems and HF radio transceivers to forward text messages and obtain weather maps.

\subsection{NVIS communication system optimization}

For disaster relief efforts, first responders in the field will have to work with battery power and improvised antennas. Similar power and antenna limitations may apply to humanitarian projects and military field operations. Optimizing the entire NVIS communication system may substantially $(10-30 \mathrm{~dB})$ reduce the required link budget and is therefore important for these applications.

The block diagram of a MIMO NVIS communication system is given in Fig. 2. $t$ can be reduced to a diversity system by omitting the second modulator, transmitter and transmit antenna, or to a Single Input Single Output (SISO) 
Fig. 2 Block diagram of an NVIS communication system. Two propagation channels are shown, as in diversity and Multiple Input, Multiple Output (MIMO) systems. Mod. and Demod. stand for modulator and demodulator; Tx, 'Rx and 'Ant.' stand for transmitter, receiver and antenna

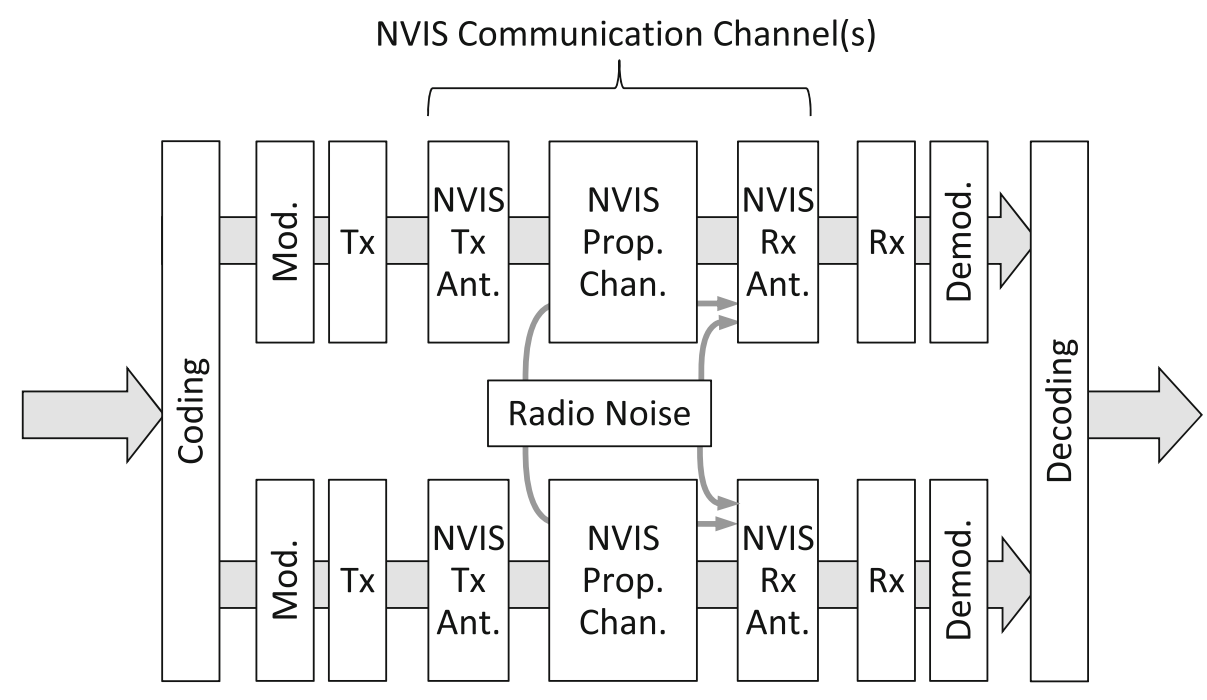

system by omitting the entire second transmit-receive chain. Antenna and propagation channel together define the propagation channel for which the modulation and coding must be optimized. Radio noise is present in the propagation channel, but also picked op directly by the receive antenna from its surroundings. System optimization requires research on antenna parameters, propagation mechanism, diversity, channel parameters, modulation techniques and coding. While specialization is needed for in-depth research in each one of these fields, their interaction is substantial and needs to be considered when studying one single aspect. For example, the chosen antenna pattern and polarization influences channel fading and time dispersion, resulting in different coding and modulation optima.

\subsection{Motivation of this review}

Up to the 1960s most of transoceanic communications were performed by HF radio systems, thanks to high altitude reflections by means of the ionosphere. Considering that some of the inventions that led to that technology stem from the 1930s, they must be considered an impressive innovation. Nowadays, satellites and transoceanic glass fiber cables for modern communications, which provide higher throughputs and can achieve good quality of service (QoS), have replaced HF communications. Recently there, however, there has been revival of the use of HF radio systems because todays technology provides affordable HF radio systems, when in the past their cost was significantly higher. Therefore, there is a renewed scientific interest in NVIS antennas, propagation and modem design following these technological advances, which also finds its way into standardization. HF radio communication systems and NVIS systems in particular, remain important for the specific applications previously depicted, where conventional communications systems cannot provide service or have ceased operation due to unfortunate events.
Remote sensing, defense applications, emergency communications when the infrastructures are down or connecting isolated places in developing countries, where modern communications systems cannot be deployed, are some of the applications on which NVIS HF communications are focused today.

It is therefore important to have a good overview of the research done in each of these fields. This reference collection and organization has never been done before exhaustively; firstly, it requires coverage of all the different research areas that NVIS comprises, and secondly the long research history of this topic promises a long collection of work. For full coverage of the NVIS research area both recently published papers and papers from ninety years ago are equally necessary. In addition, because of the long research history the outcome of NVIS research is scattered over a large number of scientific fora, spread over a considerable interval in time and identified with varying keywords. Consequently, to acquire an integral overview of the field alone requires a considerable effort and time, which would be better spent on research into missing aspects that connect and augment individual pieces of research. Having experienced this process in two separate NVIS research groups motivated the authors to combine their resources and make them available to other NVIS researchers.

This article provides an overview of research relevant to NVIS communication systems, discussing the building blocks and the relations between, and provides reference to relevant publications. It will help researchers to quickly build their own NVIS library. It also identifies niche subjects within the NVIS research field, where additional research will connect and augment other research and improve the overall knowledge of NVIS propagation and related systems. It may also help to find NVIS research groups with complementary research for cooperation. 
The article is structured as follows: NVIS radio wave propagation is discussed in Sect. 2. Subsequently an overview of NVIS antenna research is given in Sect. 3. NVIS channel characterization and associated modulation and coding techniques are discussed in Sects. 4 and 5. A discussion on subjects that merit more research and some concluding remarks can be found in Sect. 6.

\section{Near Vertical Incidence Skywave Propagation}

\subsection{The ionosphere}

The radiation of the sun ionizes gasses in the upper part of the Earth's atmosphere: the ionosphere. Several ionospheric layers (regions) can be identified, each layer having its particular composition and being ionized by specific wavelengths in the solar radiation. In the F-layer atomic oxygen is ionized by absorbtion of extreme UV radiation, with a peak electron density at $175 \mathrm{~km}$ height. In the E-layer, between 90 and $150 \mathrm{~km}$ in height, $\mathrm{O}_{2}$ absorbs soft $\mathrm{X}$-ray and $\mathrm{UV}$ radiation. The $\mathrm{D}$ layer, between approximately 60 and $90 \mathrm{~km}$ height, is caused by photo-ionization of $\mathrm{NO}$ molecules by Lyman-alpha radiation [32]. The D-layer, responsible for high attenuation at the lower HF frequencies, disappears almost completely at night. By daylight, the F-layer is split into a lower F1-layer and a higher F2-layer.

\subsection{NVIS propagation}

Electromagnetic waves entering the ionosphere may be refracted back to Earth, depending on the operating frequency. A wave traveling vertically will be reflected by one of the layers when its operating frequency is lower than the critical frequency of that layer. Radio waves with a frequency above the critical frequency will pass through the layer at vertical incidence, but will be reflected at lower elevation angles [33], resulting in coverage starting at a certain distance from the transmitter, and a circular zone around the transmitter remaining without coverage, as shown in Fig. 3: the 'skip zone'. To realize a coverage area around the transmitter without such a skip zone, the operating frequency must remain below the critical frequency of the layer used [32]. The propagation mechanism is then called Near Vertical Incidence Skywave (NVIS). Both the E- and F2-layer can be used for NVIS links.

Absorption as well as radio noise being lower at higher frequencies, F2-layer NVIS links will be more energy efficient [32]. Due to the large reflection height and relatively short distances covered, elevation angles are high.

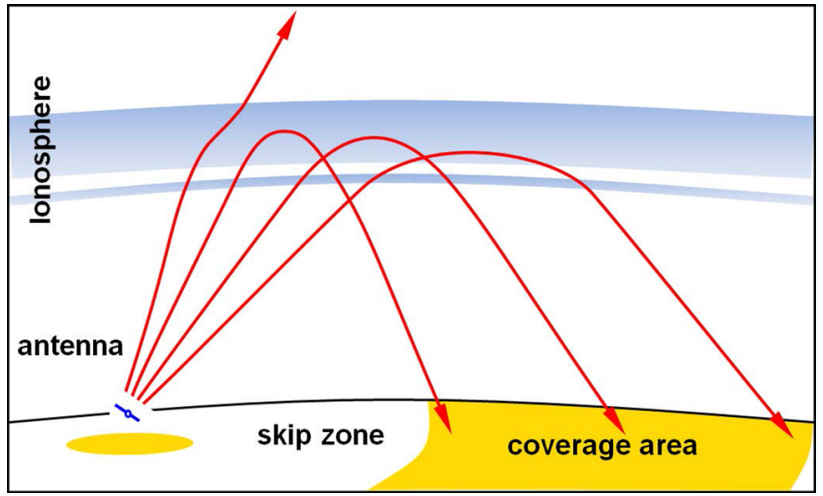

Fig. 3 Transmission above the critical frequency of the ionosphere results in a 'skip zone'. Figure from [31]

\subsection{Characteristic wave propagation}

Experiments of [11] show double reflections in the F1-layer, and double reflections in the F2-layer, as we can see in Fig. 4. Appleton mathematically proved that electromagnetic waves entering the ionosphere, under the influence of the Earth magnetic field, are split into two circularly polarized characteristic waves with opposite rotation sense, the ordinary and extraordinary wave [32, p. 82]. Appleton's magneto-ionic theory extended previous work of Maxwell and Thomson [34, pp. 404-408], who explained the polarization rotation found experimentally by Faraday in $1845[35,36]$. This magneto-ionic propagation is treated in [32,37-39]. The critical frequency of the ionospheric layer is different for each of these waves. Consequently, their path through the ionosphere is different, as can be shown with ray-tracing techniques [40,41]. The waves suffer different attenuation and show different channel characteristics, such as delay and fading patterns. The polarization of the characteristic waves, as seen when entering or leaving the ionosphere, depends on the propagation of the waves with respect to the magnetic field. In the Northern hemisphere the polarization of the ordinary wave is right-hand circular (IEEE definition) on the upward and left-hand circular on the downward path. The rotation sense is reversed in the Southern hemisphere. For mid-latitude locations (between $23.5^{\circ}$ and $66^{\circ}$ latitude) at frequencies above $5 \mathrm{MHz}$, the polarization of the characteristic waves in NVIS propagation is nearly circular. Closer to the magnetic equator the polarization approaches linear (horizontal), at the magnetic poles it is circular [32, pp. 7783].

\subsection{Diurnal variation and solar cycle}

The solar energy absorbed in the ionosphere changes with the slant of the incoming sun rays, and the ionization of the ionosphere shows a diurnal variation [42]. The critical fre- 


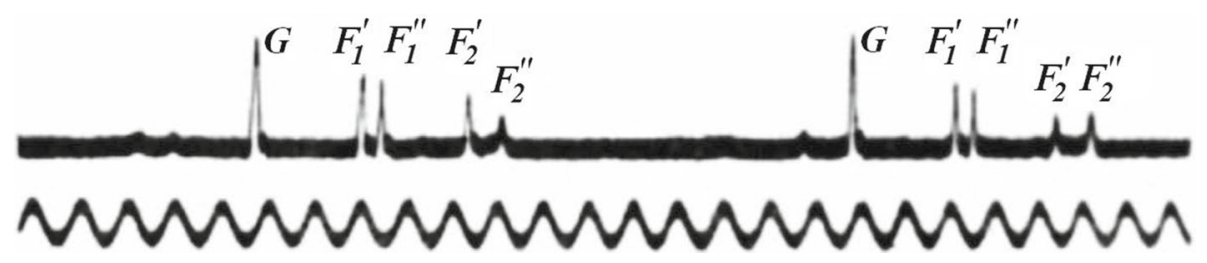

Fig. 4 NVIS measurements by Appleton and Builder. Upper trace pulses are received first via ground wave (G), then twice via the F1-layer (F1' and $\mathrm{F}^{\prime \prime}$ ) and twice via the F2-layer (F2' and F2"). Lower trace a $1115 \mathrm{~Hz}$ sine wave serving as time reference. Graph adapted from [11]

quency follows this pattern with a maximum near mid-day and a minimum in the early morning, just before sunrise. For the same reason, at mid-latitudes, the ionization follows the seasons. On top of that, the radiation of the Sun varies over time following its sidereal rotation and its 11-year sunspot cycle [32, p. 36]. To maintain NVIS propagation, a frequency has to be selected that remains below the critical frequency of the F2 layer for a larger part of the day, and a lower frequency must be used at night. However, both Walden [43] and Witvliet et al. [44] report above-the-MUF propagation at night. The latter show that the daytime characteristic wave propagation disappears at night, to be replaced by scattering with significantly lower efficiency. Research on NVIS link performance can be found in [45] for high latitudes, and in [46] for the specific case of low solar flux indices.

\subsection{Propagation prediction}

To gain insight in the present NVIS propagation, measurements from the nearest ionosonde may be used. Observing the diurnal variation of the critical frequency of the F2-layer for the ordinary wave (foF2) and extraordinary wave (fxF2 or fxI) over one or two sidereal days will give an indication of the propagation to be expected in the next few days. Johnson [47] compares measured $24 \mathrm{~h}$ NVIS link availability with VOACAP [48] simulations on several frequencies between 3 and $9 \mathrm{MHz}$ during a solar minimum. Walden [49] reports that several propagation prediction models neglect the effect of the extraordinary wave. In [50] basic recommendations and formula for ionospheric propagation prediction are provided, and in [51] a state-of-the-art ionospheric prediction model is described. Combination of ionospheric parameters measured in real time with ray-tracing software using the International Reference Ionosphere (IRI) model [52,53] makes improved short-term propagation predictions possible.

\section{NVIS antennas}

As the ITU Handbook on Emergency Telecommunications [54] states: "Time, effort and money invested in the antenna system will generally provide more improvement to communications than an equal investment to any other part of the station". Realizing this, several investigators published results on NVIS antenna optimization. Important parameters for NVIS antenna optimization are antenna diagram, polarization and bandwidth. As only high elevation angles contribute to NVIS propagation [55], optimizing the antenna diagram for these elevation angles will increase the effectively transmitted power and improve the signalto-interference ratio at reception.

This section is organized in sections on fixed, field expedient, mobile and receive antennas, each application imposing specific limitations to the antenna optimization. A section on in-situ NVIS antenna measurement is added. The section concludes with the influence of the antenna characteristics on channel parameters.

\subsection{NVIS antennas for fixed installations}

With some variation between different sources $[4,56]$ practical NVIS operating frequencies range from approximately 3-10 MHz, corresponding with wavelengths of 30-100 m. Therefore, if this entire frequency range is to be covered with high directivity and high efficiency, the antenna will be large. In fixed installations this is acceptable: the effort in mechanical engineering is balanced by a large reduction in required transmitter power, as well as an improvement immunity to interference and radio noise on reception. NVIS antennas suitable for fixed installations are the Delta antenna [57], the vertical Rhombic antenna [58, pp. 11.711.16], and the Log-Periodic Conical Spiral antenna [59]. These antennas are often used in ionosonde installations because of their frequency independent behavior. The Conical Spiral antenna provides circular polarization with high (30 dB) cross-polarization [59], its polarization sense being determined by the winding direction. In fixed military installations a vertically oriented Log-Periodic Dipole Antenna (LPDA) is also used [4, p. 48]. A derivative, the Log-Period Zig-Zag antenna, is described in [60]. Broadside arrays of multiple dipole antennas [61] may also be considered, but their frequency coverage is generally limited to one octave. The Conical Spiral antenna and the vertically Log-Periodic Dipole Antenna can be seen in Fig. 5a, b. 
(a)

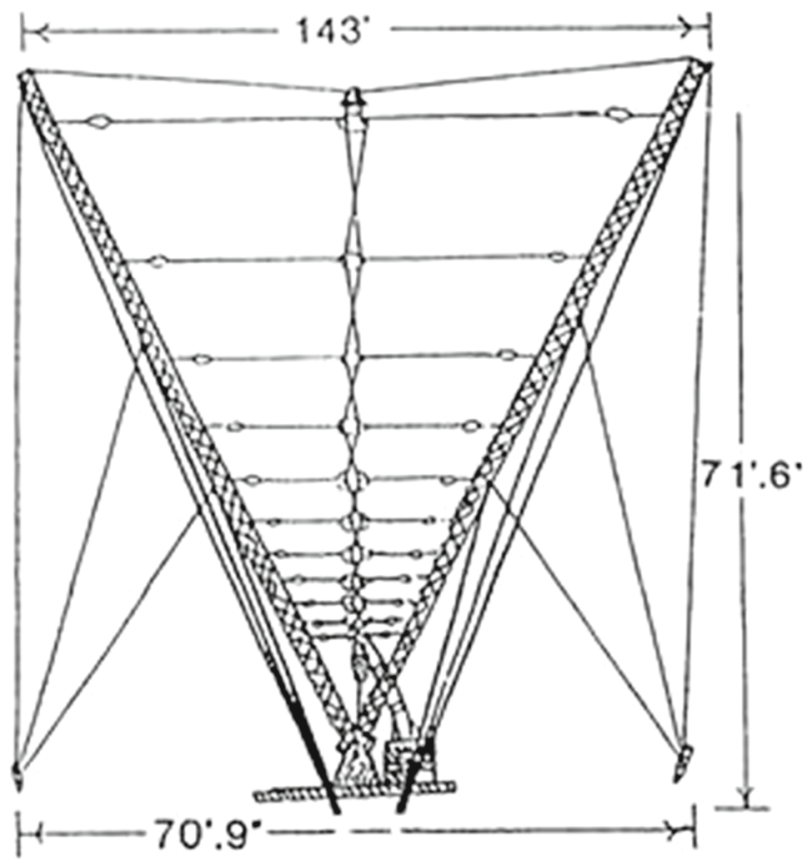

(c)

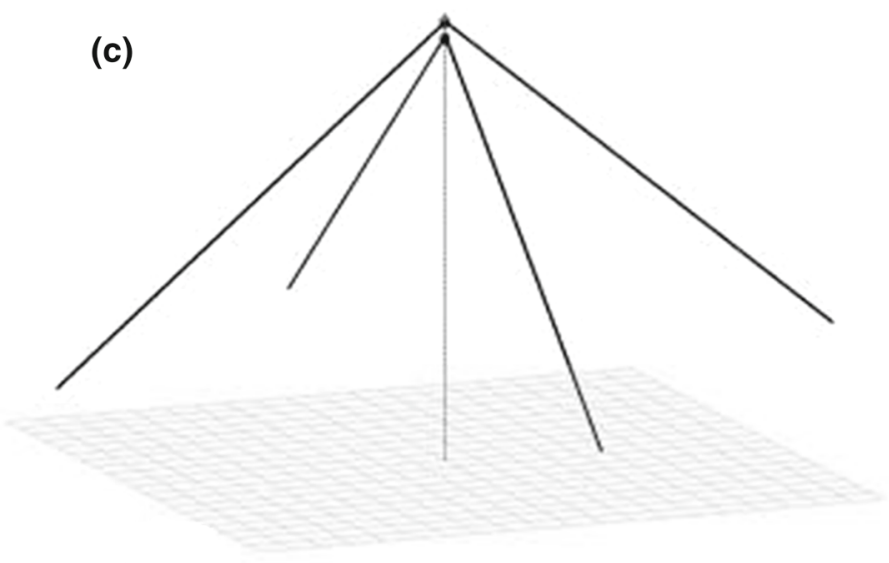

(b)

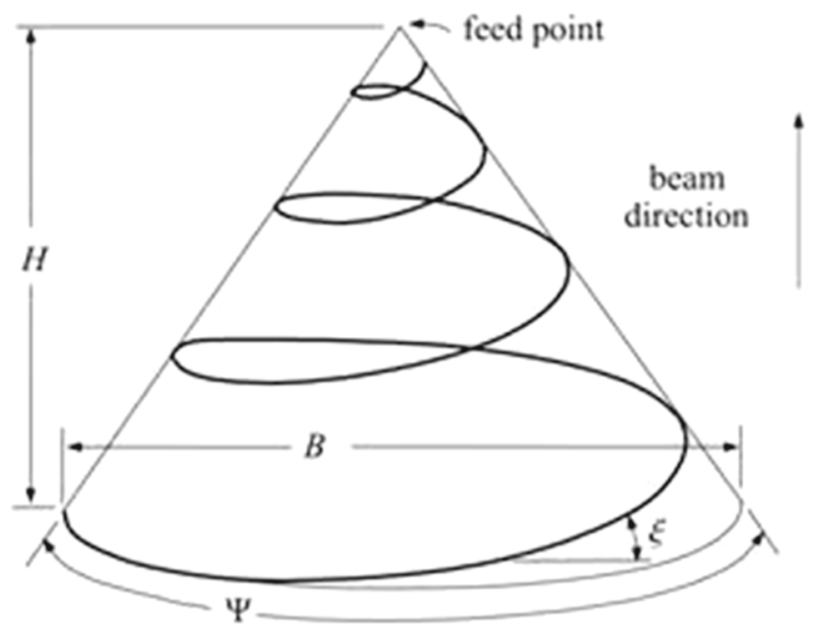

only one spiral arm shown

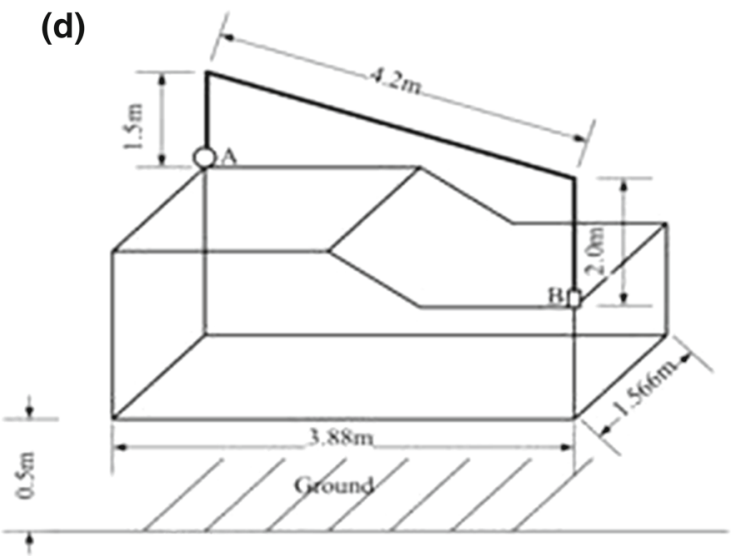

Fig. 5 NVIS antennas for fixed, portable and mobile use: a Vertical Log-Periodic Dipole Antenna (LPDA). b Conical Spiral Antenna. c Turnstile Antenna. d Mobile Car Loop Antenna. Figures taken from [4,8,59] and [19]

\subsection{Field expedient NVIS antennas}

For field expedient use, the antennas described above are not practical: their transportation is cumbersome and their installation time-consuming, in some terrain even impossible. However, simple and light wire antennas can provide good NVIS performance, alongside with the desired flexibility. E.g. a simple wire dipole antenna may exhibit an antenna gain of approximately $6 \mathrm{dBi}$ at high angles, ground reflection included [62], and such an antenna may be strung in-between trees or suspended in an 'inverted vee' fashion from lightweight extendable fiberglass or aluminum masts.
Extensive simulations and in-situ antenna pattern measurements on simple wire dipoles, Inverted L [4, p. 50] and end-fed slanted wire antennas have been performed in [62]. This research included the influence of vegetation, antenna height and the Earth's magnetic field in California, USA and in Thailand.

\subsection{NVIS antennas for mobile use}

The size limitations of antennas for moving vehicles introduce specific problems concerning radiation diagram and efficiency. Vertical whip antennas on vehicles perform badly 
in NVIS, as their radiation pattern shows a pronounced minimum at high elevation angles. Measured antenna gain at these angles range from $-35 \mathrm{dBi}$ at $4 \mathrm{MHz}$ to $-10 \mathrm{dBi}$ at $8 \mathrm{MHz}$ [63]. Tilting the whip over the vehicle will only decrease the performance [19], and tilting the whip away from the vehicle is generally not possible when on the move. In [5] a car-mounted vertical half loop antenna is described, using capacitance loading to achieve an NVIS antenna gain between -12 and $-10 \mathrm{dBi}$ from 3 to $8 \mathrm{MHz}$. This antenna is depicted in Fig. 5d. Similar NVIS loop antennas may be designed for transport aircraft [20], helicopter [64] and ships. Due to its increased size, the shipboard loop in [65] achieves an NVIS antenna gain between -1 and $+4 \mathrm{dBi}$ between 2 and $7 \mathrm{MHz}$. In helicopters, the antenna designer has to prevent unwanted modulation of the signal by the rotating rotors $[64,66]$.

\subsection{Antennas for NVIS reception}

Most of the publications presume that the same antenna is used for both transmission and reception. This is not necessarily the best solution. For reception the average directivity over the NVIS elevation angles is more important than the antenna gain [67, pp. 766-767] [31, pp. 137-143]. As HF reception is limited by the ambient electromagnetic noise or radio noise [68] rather than by the receiver sensitivity, absolute gain is less important than discrimination between the wanted signal on one hand, and radio noise and interference on the other. Discrimination of the receive antenna diagram between the NVIS elevation angles and angles at which most interference and ambient noise arrives [69] will significantly improve reception. While unsuitable for transmission, compact active antenna elements may provide maximum (i.e. ambient noise limited) sensitivity [70]. Directive arrays may be composed of several such active antenna elements and spatial filtering can be used to further improve the signal-tonoise ratio on reception [71].

\subsection{NVIS antenna pattern optimization}

For the optimization of NVIS antennas, often only the antenna gain at zenith angles is considered. However, on the desired coverage area size and the ionospheric reflection height, the average directivity in a range of spatial angles must be considered [31, p. 137]. For that purpose, graphs relating elevation angle to great circle distance are provided in $[32,72]$ and their dependency on the operating frequency and the sunspot number is shown in [31, pp. 132-135].

\subsection{In-situ NVIS antenna measurement}

Due to their size, NVIS antennas cannot be measured in anechoic rooms. This is also true for the (smaller) mobile antennas, as their supporting platform is an integral part of the radiating structure. Also the influence of the ground underneath the antenna installation is not negligible and has to be included in the measurement. However, in-situ measurement of antenna pattern and relative gain can be used for antenna evaluation, using a small transmitter transported by helicopter [73], airplane [74], tethered balloon [5] or remote controlled octocopter drone [75]. Due to the fast and deep fading imposed by ionospheric propagation, comparison of antenna performance using real-live NVIS transmitters is only possible when specialized techniques are used, such as described in [31, pp. 139-140]. In [17] an ionosonde is used to compare the antenna gain of two antennas at zenith angle using pulsed measurements.

\section{NVIS channel characteristics}

In this section NVIS channel sounding and modelling is discussed, including their variation with latitude. Several experiments have been conducted by Burgess and Evans [76] and Tooby et al. $[77,78]$ to characterize the NVIS channel in the United Kingdom. Their results can be used to simulate the influence of the channel, enabling performance testing of modulation and coding systems without on-air measurements [79]. Channel simulations also enable comparison of modulation and coding systems in identical circumstances, which is not possible with real propagation.

\subsection{NVIS channel sounding}

In order to design the modulation and coding system that minimises the Bit Error Rate (BER) and maximizes the throughput of an ionospheric link, a sounding system covering the entire HF band was designed in [80] and improved in [81], in this case for a long distance link. Real data from a Lowell ionosonde [82] has been used by Hervas et al. [56] to characterize the NVIS channel. This data can be used to trade-off BER and bit rate when designing modulation and coding techniques for an HF NVIS channel. Other research uses Digital Radio Mondiale (DRM) broadcast signals [83] and radio noise measurements to calculate the parameters with which the best channel availability is obtained [84,85]. The latter discusses medium-wave NVIS propagation, which has different characteristics than HF NVIS propagation. Previous research of Tooby [77] used a chirp sounder to measure the signal-to-noise ratio (SNR) and multipath characteristics between 1998 and 1999.

\subsubsection{Fading}

Ionospheric propagation is prone to signal fading. Figure 6 shows slow fading caused by changing ionospheric reflec- 
Fig. 6 NVIS measurements, showing fast deep multipath fading superimposed on slower flat fading. Figure taken from [8]

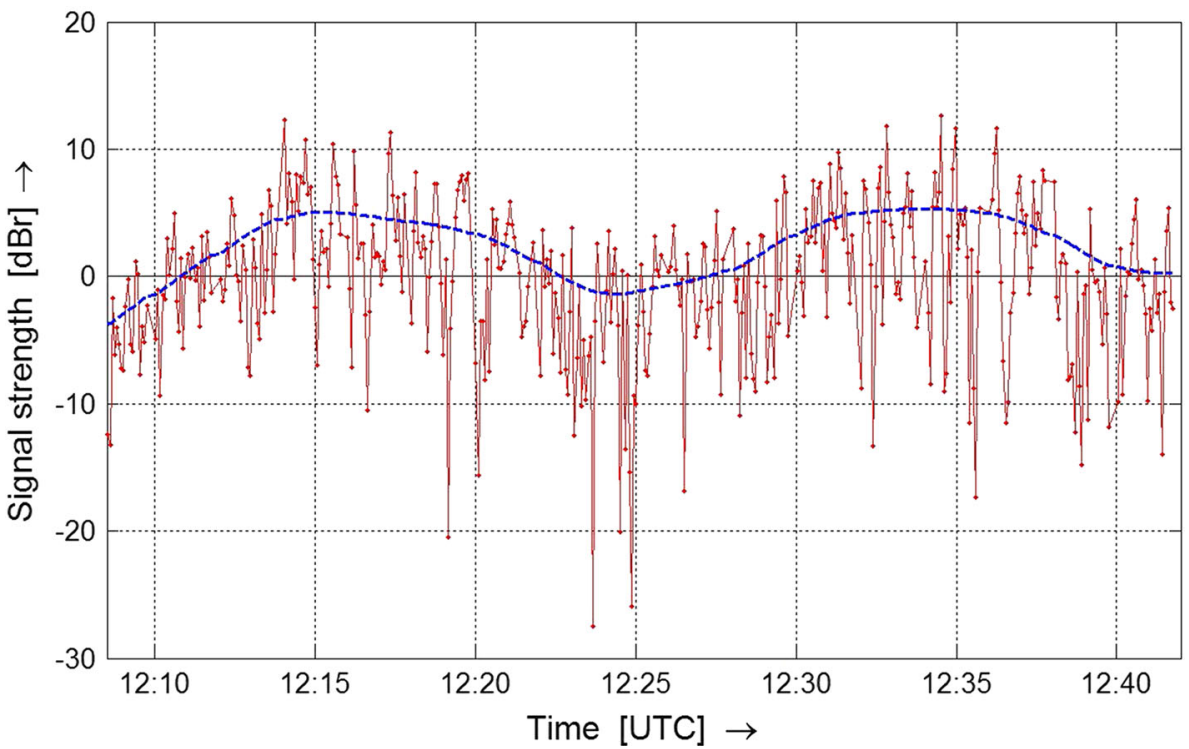

tivity and absorption on a timescale of tens of minutes, with superimposed fast (seconds) and deep (down to - 30 dB) multipath fading, caused by interference between signals arriving via ionospheric paths of different length [86]. For the design of communication systems, knowledge of the mean value of the received signal is insufficient: signal fading has a pronounced effect on the data reception. An extra signal margin has to be reserved for the fading minima, the 'fading margin', and the coding has to be adapted to allow for temporary symbol loss. McNicol [87] discusses fading on vertical incidence signals between 2 and $6 \mathrm{MHz}$.

Fading time series are usually studied and modeled using stochastic processes [88,89]. It is assumed that the fading generated by the composition of multiple ionospheric echoes can be represented as the sum of the specularly reflected signals plus a random component; this is modelled by the Nakagami-Rice distribution [90]. If the random component dominates, this probability function approaches a Rayleigh distribution [91]. According to Davies [32, p. 237], short observations of ionospheric fading on long-distancxe links will reveal Rayleigh fading, while longer observations will show a log-normal signal distribution. Burgess [76] shows that NVIS propagation conditions may exist also in which fading is almost completely absent.

\subsubsection{Doppler spread}

Not only the depth of the fading is important, but also its periodicity. Fading rates are often expressed in terms of the autocorrelation of the times series. This autocorrelation measures the time difference by which a point in the time series is decoupled from its neighbors. This time difference measured by autocorrelation is usually called 'coherence time' [92]. The coherence time has a direct relationship with the
Doppler spread of the channel [92]. Channels with large Doppler spread have signal components that change in phase over time, since the fading depends on whether the addition of components is constructive or destructive; these channels have a short coherence time. The coherence time has to be taken into account when choosing the symbol time of the modulation, to assure that the channel has an approximately constant response during the symbol.

\subsubsection{Delay spread}

The delay spread is a measure of the amount of multipath propagation in a communications channel [92]. In general, it is interpreted as the difference between the time of arrival of the earliest significant multipath component and the time of arrival of the latest significant multipath component. It is usually obtained by measuring the power delay profile of the channel [93]. Figure 7 shows both Doppler and Delay Spread of a high latitude path in Scandinavia [94]. The delay spread may cause Inter Symbol Interference (ISI). When designing a modulation system, the symbol duration must be long enough-usually 10 times the delay spread - so that an ISIfree channel is obtained [95]. Delay spread in the time domain is linked to 'coherence bandwidth' in the frequency domain, which is the is a statistical measure of the range of frequencies over which the channel passes all spectral components with approximately equal gain and linear phase [95].

\subsection{NVIS channel modelling}

For many years, the model of Watterson et al. [96], also described in ITU-R Rec. F.1487 [97], has been the standard ionospheric channel model for analysis and performance evaluation of HF communication systems. While this model 


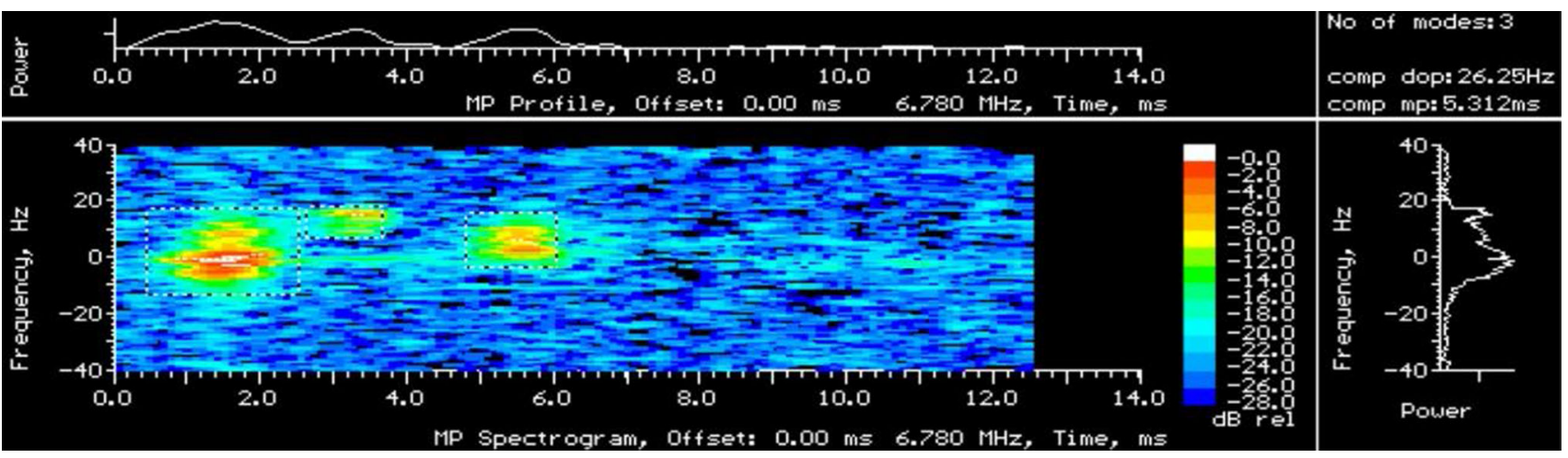

Fig. 7 Doppler and delay spread on a high latitude path in Scandinavia, measured with Doppler And Multipath SOunding Network (DAMSON). Figure adapted from [94], a measurement on a $180 \mathrm{~km}$ NVIS link between Harstad (Norway) and Kiruna (Sweden)

properly simulates the signal perturbations such as signal fading, Doppler and delay spread occurring in an interval of a few seconds, it does not represent the variations of the channel quality, especially the SNR, occurring in longer intervals in the range of a few seconds to $10 \mathrm{~min}$. In [98], both longhaul and NVIS measurements are performed to model the SNR variations to improve the Watterson model. Some work can be found on the study and modelling of the channel characteristics using polarization diversity techniques to improve system performance [56]. Finally, measurements on broadcast signals in the medium wave (MW) band are used to improve the accuracy of the channel predictions for network planning in that band [84,99], providing the first channel model for NVIS in the medium wave band [100]; a tap delayed line (TDL) model based upon the field measurements.

\subsection{NVIS channel characteristics as function of the latitude}

In several propagation reference books $[32,38,101]$ detailed explanations about the physical phenomena in the ionosphere are presented. In terms of HF communications, the ionosphere study is divided into three zones: (i) polar zone (latitudes greater than $66^{\circ}$ ), (ii) mid latitudes (between $23.5^{\circ}$ and $66^{\circ}$ ) and (iii) equatorial zone (between $23.5^{\circ} \mathrm{N}$ and $23.5^{\circ} \mathrm{S}$ ) [102], each of them with its own particularities.

Wagner and Goldstein [45] are among the first to characterize the performance of the high-latitude ionospheric NVIS channel. Warrington et al. [103] and Jodalen et al. [104], amongst others, also provide a thorough channel analysis for high latitudes NVIS paths. Jodalen et al. [104] presents the results from a propagation experiment over two NVIS paths in Northern Scandinavia using Doppler and Multipath SOunding Network (DAMSON) [105], with the aim of correlating the results of both paths. Warrington et al. [103] presents direction finding measurements in Norway and Swe- den, to better understand the directional characteristics of HF signals reflected in the high-latitude ionosphere. Lossman et al. [106], who performed tests in the Baltic region, concluded that transmit frequencies greater than $5 \mathrm{MHz}$ show higher effective data speeds due to the fact that man-made noise is mostly concentrated between 2 and $5 \mathrm{MHz}$.

In [77], the quality of mid-latitude NVIS channels is measured, evaluating SNR and multipath data. Hervas et al. [56] performs mid-latitude NVIS channel soundings using a Lowell Digisonde [82], obtaining information about Doppler and multipath delay spread, and the best possible transmission frequency as function of the hour of the day. Austin [15] describes the performance of the equatorial ionosphere, and finally, in [105] channel sounding with the DAMSON system was used to evaluate polar, mid-latitude and equatorial propagation channels for the use of $12 \mathrm{kHz}$ wide HF broadcast systems operating on fixed frequencies and requiring high availability.

\section{Modulation and coding for the NVIS channel}

This section consists of two blocks. The first focuses on mapping different data transmission methods for NVIS technology and enumerating several physical layer proposals responding to the needs of each of the communication situations. The second block gives light on the use of diversity and MIMO techniques to improve data transmission throughput using time, space, and frequency or polarization diversity.

The aim of this section is to give the reader an idea of the map of previous scientific work performed related to NVIS modulation and coding. It not meant to be an exhaustive comparison between the different modulations or codes, since they are too different from each other to be compared one by one; the intention is not to conclude that there is one better than the others are, since each responds to their circumstances. The reader will be able to find references that are of 
interest reading a brief description of each; also a comparative table of the basic parameters in terms of SNR, bandwidth and throughput is presented at the end of the section, gathering most of the proposals.

\subsection{Data transmission methods for NVIS}

In the bibliography, several different physical layer designs can be found that are adapted to the parameters that are specific to HF propagation. There are no special recommendations for NVIS links, all the HF recommendations and standards cover both long-haul and short distance communication.

\subsubsection{ITU recommendations}

Several ITU recommendations contain information on fading and its impact on modulation [86], delay spread evaluation [93] and modem test [97]. These documents are developed by the Radiocommunication Sector, and are informative only, but give the first keys of the implementation to the designer.

\subsubsection{Military HF data transmission protocols}

Some of the HF Data Transmission Protocols (e.g. STANAG 4415, MIL-STD $118110 \mathrm{C}$ and DRM) are designed to work for both NVIS and oblique incidence propagation, and are optimized for data transfer in the presence of fading, multipath and noise. In [107], the link availability of two modems (one compatible with STANAG 4415 and 4285, and the second only with STANAG 4285) is compared with Morse code and voice traffic. Davies [108] contains measurements of throughput in relation to SNR for STANAG 4539 [109] over several distances, to evaluate the effects of low SNR and variable channel conditions, taking into account advanced waveforms, coding and protocols.

In [110] two alternative channel access technologies are tested and compared in wireless mesh networks: Automatic Link Establishment (ALE) and fixed-frequency MAC protocols. These are also described in the book of Johnson et al. [111]. In [112] we find measurements on a MIL-STD-188110C [113] link over distances of up to $160 \mathrm{~km}$, providing the users with bit rates up to $9.6 \mathrm{kbps}$ in $6-9 \mathrm{kHz}$ RF bandwidth. The standard gives the option of selecting bandwidth and modulation, and includes wideband modulation with up to $24 \mathrm{kHz}$ bandwidth with elaborate coding and interleaving.

\subsubsection{HF broadcasting protocols}

Digital Radio Mondial (DRM), an international radio broadcasting standard launched in 2003, solves fading and phase distortion problems associated with shortwave AM broadcasting and offers both increased audio quality and band- width [85]. Spreading the information in frequency and time over multiple carriers using OFDM modulation makes the system less prone to selective fading. Coding and interleaving are implemented and their parameters can be selected to match the expected propagation channel. An option for NVIS is included. In the thesis of Losada [85] a study of the application of DRM is detailed, evaluating the statistical performance of the modem in different environments (Doppler spread, delay spread, interference), but it must be noted that the empirical results come from medium-wave NVIS experiments.

\subsubsection{Commercial radio protocols}

Commercial HF radio modem protocols such as PacTor IV claim to achieve $5.5 \mathrm{kbps}$ in $2.4 \mathrm{kHz}$ RF bandwidth at $17 \mathrm{~dB}$ SNR and to maintain the link down to $-21 \mathrm{~dB}$ SNR [114]. PacTor has proven successful in disaster relief communication and is used for radio mail and weather map transfer for sea sailing yachts. A scientific comparison with other protocols is provided by ITU-R Recommendation F.339 $[115,116]$.

\subsubsection{Experimental physical layer definitions}

Hoult et al. [9] present a study to achieve $16 \mathrm{kbps}$ in a standard $3 \mathrm{kHz}$ wide HF channel, to improve on the 2.4 or $4.8 \mathrm{kbps}$ modems that were available at the time. It uses an NVIS channel instead of a VHF link, which could not be used due to the accidented terrain. Several other studies were done to improve the effective bitrate of modems used in one-hop ionospheric propagation and NVIS propagation [107]. Johnson [110] details modem characteristics for a wireless mesh network for NVIS propagation, long-haul skywave and surface wave signals.

In [117] there is a study of several modulation techniques tested over a distance of $160 \mathrm{~km}$ on frequencies between $2.8 \mathrm{MHz}$ and $9.4 \mathrm{MHz}$, presenting measured SER (Symbol Error Rate) values for data rates from 4.3 to $20.6 \mathrm{kbps}$. More details of this work can be found in Christofi's thesis [118]. Finally, [119] presents over the air transmissions results obtained with a multi-narrow band HF modem operating over non-contiguous $3 \mathrm{kHz}$ bands spread over a $200 \mathrm{kHz}$ wide subband.

\subsection{Diversity and MIMO}

Reducing fading and increasing throughput on an $\mathrm{HF}$ channel can also be realized using diversity techniques. Frequency diversity, time diversity, antenna (spatial and polarization) diversity and adaptive beamforming to suppress unwanted multipath were originally invented in the early 1930s, and implemented in intercontinental HF radio communication 
Table 1 An overview of the net throughput, bandwidth and SNR in a fading channel of several transmission systems

\begin{tabular}{|c|c|c|c|c|c|c|}
\hline Protocol & Modulation & Net datarate & $\mathrm{SNR}(\mathrm{dB})$ & BW (kHz) & $\operatorname{SNR}(3 \mathrm{kHz})[\mathrm{dB}]$ & Source \\
\hline PacTor IV (speed 1) & 2 tone chirp & 47 bps & -22 & 2.4 & -23 & {$[114]^{*}$} \\
\hline MIL-STD-188-110A & 8-PSK & 75 bps & 3 & 3 & 3 & [116] \\
\hline Pactor II (speed 4) & 2-tone DPSK & $150 \mathrm{bps}$ & 8 & 0.5 & 0 & [116] \\
\hline Pactor II (speed 6) & 2-tone DPSK & 300 bps & 28 & 0.5 & 20 & [116] \\
\hline MIL-STD-188-110A & 2-tone 8-PSK & $2.4 \mathrm{kbps}$ & 18 & 3 & 18 & [116] \\
\hline MIL-STD-188-110B & QPSK & $3.2 \mathrm{kbps}$ & 15 & 3 & 15 & [116] \\
\hline PacTor IV (speed 10) & 18-tone 32-QAM & $5.5 \mathrm{kbps}$ & 14 & 2.4 & 13 & {$[114]^{*}$} \\
\hline Experimental & 56-tone 256-QAM & $8 \mathrm{kbps}$ & 23 & 3 & 23 & [9] \\
\hline MIL-STD-188-110B & 64-QAM & $9.6 \mathrm{kbps}$ & 33 & 6 & 36 & [116] \\
\hline Experimental & 256-QAM & $16 \mathrm{kbps}$ & 35 & 3 & 35 & [9] \\
\hline Experimental & 16-QAM MIMO & $60 \mathrm{kbps}$ & 19 & 25 & 28 & [122] \\
\hline MIL-STD-188-110C & 256-QAM & $64 \mathrm{kbps}$ & 20 & 24 & 29 & [112] \\
\hline
\end{tabular}

As measurement methods are not always described in full and differ from one researcher to the other, these values must be taken as indicative. The items with * are unverified manufacturer specifications only

systems $[120,121]$. At the technological level of that era this required great ingenuity and considerable expenses. Today's $\mathrm{RF}$ and signal processing hardware allows compact and cheap realization of elaborate diversity systems, promising substantial reductions in required link budget. Diversity system may be integrated in the coding software on both ends of the link (MIMO), in selection software after the demodulator (selection diversity), or in the baseband of the receiver (e.g. optimum ratio combining). Enserink [122] proposes a MIMO solution doubling the net data throughput from 30 to $60 \mathrm{kbps}$ in a $25 \mathrm{kHz}$ channel. Using modern means to generate and receive dual circular polarization [123], characteristic wave propagation may be used to provide increased diversity gain $[124,125]$. NVIS characteristic wave MIMO systems have been proposed by Ndao et al. [126].

An overview of the net throughput, bandwidth and SNR in a fading channel of several transmission systems is given in Table 1 for comparison. To compare the necessary received signal strength for systems with a different bandwidth, the SNR has been calculated in an equivalent bandwidth of $3 \mathrm{kHz}$ also. As measurement methods are not always described in full and differ from one researcher to the other, these values must be taken as indicative.

\section{Discussion and conclusions}

A large amount of research has already been done on individual components of the NVIS radio communication system, but several subjects remain that merit investigation, to augment or interconnect the existing research. In this section, we have identified the niche subjects that merit future investigation. Both research in antennas and propagation as well as in modulations and channel coding will lead NVIS research to the next stage of evolution using todays technology, the first due to the polarization diversity study, which will increase the benefits of the ionospheric channel propagation, and the second one to improve the throughput and the quality of service of the communications. References to recent work on the topic are added to record the research niches that are being developed by different research groups that presently perform research in those fields.

\subsection{Antennas and propagation}

Empirical research into the nature of polarization fading, together with measures to reduce this fading. Documented observations of the presence of multipath fading in a single hop single layer NVIS propagation path-with polarization fading excluded - would trigger new research on the nature of fading mechanisms within a single layer.

Using the definitions of NVIS antenna gain and NVIS directivity given in [31], comparison of a large number of NVIS antenna types, preferably for several coverage area sizes, and accompanied by their optimum dimensions and optimum installation heights would be a valuable asset as reference material for NVIS antenna selection. Verification of theoretical and simulated antenna optimizations under live NVIS propagation conditions are desirable to validate these optimizations.

Experiments with antennas with polarization matched to the characteristic waves in the ionosphere could bring new insights and applications. Compact antennas to produce adapted or adaptive polarization would be an asset for such experiments. Research into arrays of small active antenna elements providing maximum NVIS directivity or spatial filtering could significantly contribute to enhanced NVIS reception in areas where man-made noise is dominant. 
Several research groups are already working on those future trends in NVIS propagation and antenna design nowadays. In [43], Walden describes the findings associated with an experiment conducted at $5 \mathrm{MHz}$ in the United Kingdom and studies the high frequency Near Vertical Incidence Skywave Propagation; while in [44] Witvliet et al. study deeply the impact of a Solar X-Flare on NVIS characteristic wave propagation, giving details of the daytime characteristic wave refraction and of the nighttime scattering. Both authors report on nighttime above-the-MUF propagation. Finally in [127], Ignatenko et al. detail a wide-band mobile HF antenna design for NVIS applications.

\subsection{Channel modulation and coding}

Channel simulators for F2-layer and E-layer NVIS links for several bandwidths are helpful to compare and improve modulation and coding protocols. These models should incorporate selectable and combined ordinary and extraordinary wave channels for the design of diversity and MIMO systems. Comparison of a large number of standardized HF communication protocols over identical NVIS channels would allow comparison and analysis of the effectiveness of different solutions in modulation, coding, and adaptive behavior and the efficiency of their spectrum use. Open (nonproprietary) modulation, coding and data transfer protocols are needed, to encourage further development by third parties. Protocols designed to achieve consistent high data rates in the complex NVIS channels are much sought after, but high data rates are not always priority. Research into low speed protocols achieving highly reliable data transfer with acknowledgment at very low SNR are invaluable to transfer lifesaving information when circumstances (antenna situation, battery power, propagation) are unfavorable. Diversity and MIMO systems may increase data throughput at low SNR values. Research into efficient solutions for conversion and digital transmission of narrowband $(300 \mathrm{~Hz}-3 \mathrm{kHz})$ voice would encourage the replacement of present low-cost applications using analog modulation. In this direction, low cost flexible all-digital hardware platforms are needed for the implementation and testing of open modulations and codes, and for ad-hoc designs for specific applications [128].

\subsection{Interaction of building blocks}

Research into the interaction of building blocks such as antenna, propagation, modulation system and coding, may prevent that isolated optimization of single blocks leads to a suboptimal overall design.

\subsection{Conclusions}

An overview is given of the main building blocks of an NVIS radio communication system and specialized research into each of these building blocks. References are provided to a large cross section of the existing literature on NVIS radio communication systems, to provide new researchers in this field with an initial NVIS library, without pretending to be exhaustive. Investigators are encouraged to contribute to NVIS research, especially in the areas indicated in the previous Section, to contribute to increased reliability of NVIS emergency communication systems.

Acknowledgements Rosa Ma Alsina-Pagès would like to thank the Secretaria d'Universitats i Recerca del Departament d'Economia i Coneixement (Generalitat de Catalunya) under Grant ref. 2014-SGR0590 .

Open Access This article is distributed under the terms of the Creative Commons Attribution 4.0 International License (http://creativecomm ons.org/licenses/by/4.0/), which permits unrestricted use, distribution, and reproduction in any medium, provided you give appropriate credit to the original author(s) and the source, provide a link to the Creative Commons license, and indicate if changes were made.

\section{References}

1. Straw, R. D. (2005). What's the deal about NVIS? QST, 12, 38-43.

2. Lindquist, R. (2005). The Katrina Chronicles. QST, 11, 43-48.

3. Ewald, S., \& Lindquist, R. (2006). The Katrina Chronicles 2. QST, 2, 50-53.

4. Fiedler, D. M., \& Farmer, E. J. (1996). Near vertical incidence skywave communication: Theory techniques and validation. Sacramento: Worldradio Books.

5. Austin, B. A., \& Murray, K. P. (1988). The application of characteristic-mode techniques to vehicle-mounted NVIS antennas. IEEE Antennas and Propagation Magazine, 40(1), 7-30.

6. Rufenach, C. L., \& Hagn, G. H. (1966). Comparison of C-2 ionospheric sounder data with frequency predictions for short-range communication with man-pack transceivers in Thailand, Spec. Techn. Rep. 15, Stanford Research Institute, Menlo Park, CA, USA.

7. Perlman, S. (1970). High frequency SAFOC digital data link tests, R \& D Techn. Rep. ECOM-3341, ECOM US Army Electronics Command, Ft. Monmouth, NJ, USA.

8. Witvliet, B. A., Van Maanen, E., Petersen, G. J., Westenberg, A. J., Bentum, M. J., Slump, C. H., et al. (2015). Measuring the isolation of the circularly polarized characteristic waves in NVIS propagation. IEEE Antennas and Propagation Magazine, 57(3), 120-130.

9. Hoult, N. S., Whiffen, J. R., Tooby, M. H., \& Arthur, P. C. (2000). $16 \mathrm{kbps}$ Modems for the HF NVIS Channel. In Presented at HFRST (pp. 317-321) .

10. Appleton, E. V., \& Bartlett, M. A. F. (1925). On some direct evidence for downward atmospheric reflection of electric rays. Proceedings of the Royal Society of London, 109(752), 621-641.

11. Appleton, E. V., \& Builder, G. (1933). The ionosphere as a doublyrefracting medium. Proceedings of the Physical Society, 45(2), 208-220.

12. Heising, R. A. (1940). Radio extension links to the telephone system. Bell System Technical Journal, 19(4), 611-646. 
13. Maunsell, H. I., \& Stafford, J. W. (1964). Telstar design and construction and early results of the scientific space experiments. IEEE Transactions on Communication and Electronics, 83(70), 27-37.

14. Mervin, K. J., Radley, G., Gilman, G. W., \& Halsey, R. J. (1955). A transatlantic telephone cable. Bell System Technical Journal, 102(2), 117-130.

15. Austin, B. A. (2000). Near vertical incidence skywaves in world war II: An historical perspective, HF Radio Systems and Techniques. Guidford.

16. Ray, W. A., Barker, G. E., \& Martensen, S. S. (1966). Full-scale pattern measurements of simple HF field antennas in a US conifer forest. Spec. Tech. Rep. 25, Stanford Research Inst., Menlo Park, CA, USA.

17. Hagn, G. H. (1973). On the relative response and absolute gain toward the zenith of HF field-expedient antennas: Measured with an ionospheric sounder. IEEE Transactions on Antennas and Propagation, 21(4), 571-574.

18. Strangeways, H. J. (2006). Estimation of signal correlation at spaced antennas for multimoded ionospherically reflected signals and its effect on the capacity of SIMO and MIMO HF links. In Presented at IRST, London, UK (pp. 306-310).

19. Austin, B. A., \& Liu, W. C. (2002). Assessment of vehicle mounted antennas for NVIS applications. IEE ProceedingsMicrowaves, Antennas and Propagation, 149(3), 147-152.

20. Cummings, N. P. (2005). Design and analysis of a pattern selectable airborne HF antenna. In Presented at ACES (pp. 499502). Hawaii: Honololu.

21. Kwasinski, A., Weaver, W. W., Chapman, P. L., \& Krein, P. T. (2006). Telecommunications power plant damage assessment caused by Hurricane Katrina-Site survey and follow-up results. In: Presented at INTELEC. Providence, RI, USA.

22. Mikami, T., Shibayama, T., \& Esteban, S. (2012). Field survey of the 2011 Tohuku earthquake and Tsunami in Miyagi and Fukushima Prefectures. Coastal Engineering Journal, 54(1), 117-130.

23. Bodson, D. (1992). When the lines go down. IEEE Spectrum, 29(3), 40-44.

24. Sims, B. (2007). The day after the Hurricane: Infrastructure order, and the New Orleans Police Department's Response to Hurricane Katrina. Social Studies of Science, 37(1), 111-118.

25. Comfort, L. K. (2006). Cities at rtisk: Hurricane Katrina and the Drowning of New Orleans. Urban Affairs Review, 41(4), 501-516.

26. Maitland, D. (1984). The missing link. International Telecommunication Union, Geneva: Report of the International Commission for World-Wide Telecommunications Development.

27. Ayeni, V., \& Milward-Oliver, G. (2005). Maitland+20: Fixing the missing link. Bradford on Avon: Anima Centre.

28. Linden, L. F. (2004). Winlink 2000 in the Jungle. QST, 11, 42-45.

29. Le Masson, J., Erhel, Y. M., \& Mamane, A. R. (2012). Simulated performance of the STANAG $4538 \mathrm{HF}$ transmission standard. In Presented at IRST, UK: York.

30. Adorian, P., \& Dickinson, A. H. (1952). High frequency broadcast transmission with vertical radiation. Journal of the British Institution of Radio Engineers, 12(2), 111-116.

31. Witvliet, B. A., Van Maanen, E., Petersen, G. J., Westenberg, A. J., Bentum, M. J., Slump, C. H., et al. (2015a). Near vertical incidence skywave propagation: Elevation angles and optimum antenna height for horizontal dipole antennas. IEEE Antennas and Propagation Magazine, 57(1), 1-18.

32. Davies, K. (1990). Ionospheric radio. Exeter: Peter Peregrinus.

33. Martyn, D. F., Cherry, R. O., \& Green, A. L. (1935). Long distance observations of radio waves of medium frequencies. Proceedings of the Physical Society, 47(2), 340-351.

34. Maxwell, J. C. (1873). A treatise on electricity and magnetism, part II. Oxford: Clarendon Press.
35. Faraday, M. (1845). On the magnetization of light and the illumination of magnetic lines of force. Philosophical Transactions, 1 , $1-30$.

36. Knudsen, O. (1976). The Faraday Effect and Physical Theory 1845-1873. Archive for History of Exact Sciences, 5(3), 235-281.

37. Ratcliffe, J. A. (1962). The magneo-ionic theory and its application to the ionosphere. London: Cambridge University Press.

38. Budden, K. G. (1985). The propagation of radio waves. London: Cambridge University Press.

39. Rawer, K. (1993). Wave propagation in the ionosphere. Dordrecht: Kluwer Academic.

40. Reilly, M. H. (1991). Upgrades for the efficient three-dimensional ionospheric ray tracing: Investigation of HF near vertical incidence sky wave effects. Radio Science, 26(4), 971-980.

41. Reilly, M. H. (2000). Ray trace calculation of ionospheric propagation at lower frequencies. Radio Science, 41(5), 1-6.

42. Chapman, S. (1939). The atmospheric height distribution of bandabsorbed solar radiation. Proceedings of the Physical Society, 51(1), 93-109.

43. Walden, M. C. (2016). High-frequency near vertical incidence skywave propagation. IEEE Antennas and Propagation, 58(6), 16-28.

44. Witvliet, B. A., van Maanen, E., Petersen, G. J., \& Westenberg, A. J. (2016). Impact of a solar X-flare on NVIS propagation. IEEE Antennas and Propagation Magazine, 58(6), 29-37.

45. Wagner, L. S., \& Goldstein, J. A. (1995). Channel spread parameters for the high-latitude, near-vertical-incidence-skywave $H F$ channel: Correlation with geomagnetic activity, NRL/FR/555095-9772. Washington: Naval Research Lab.

46. Farmer, E. J. (1996). NVIS propagation at low solar flux indices. In D. M. Fiedler \& E. J. Farmer (Eds.), Near vertical incidence skywave communication: Theory, techniques and validation. Sacramento: Worldradio Books.

47. Johnson, E. E. (2007). NVIS communications during the solar minimum. In Presented at MILCOM, Orlando, Fla.

48. Perkiomaki, J. (2003-2014). HF propagation prediction and ionospheric communications analysis. www.voacap.com.

49. Walden, M. C. (2009). The extraordinary wave mode: Neglected in current practical literature on HF NVIS communications. In Presented at IRST, Edinburgh.

50. ITU. (1995). Reference ionospheric characteristics and methods of basic MUF, operational MUF and ray-path prediction. ITU-R Rec. P.434-6, International Telecommunications Union, Geneva, Switzerland.

51. Zolesi, B., \& Cander, L. R. (2014). Ionospheric prediction and forecasting. Berlin: Springer.

52. Wilkinson, P. J. (2004). Ionospheric variability and the international reference ionosphere. Advances in Space Research, 34(9), $1853-1859$.

53. Bilitza, D., McKinnell, L. A., Reinisch, B., \& Fuller-Rowell, T. (2011). The international reference ionosphere (IRI) today and in the future. Journal of Geodesy, 85(12), 909-920.

54. ITU. (2005). Handbook on emergency telecommunications. Geneva: International Telecommunications Union.

55. Black, Q. R., Wood, J. F, Jr., \& Sherill, W. M. (1995). Mode angles of arrival in the 55- to 3500-km range. Radio Science, 30(3), 693702.

56. Hervas, M., Pijoan, J. L., Alsina-Pages, R., Salvador, M., \& Altadill, D. (2013). Channel sounding and polarization diversity for the NVIS channel. In Presented at Nordic HF, Faro, Sweden.

57. Cones, H. N., Cottony, H. V., \& Watts, J. M. (1950). A 600ohm multiple-wire delta antenna for ionosphere studies. Journal of Research NBS, 44, 475-488.

58. Johnson, R. C. (1993). Antenna engineering handbook (3rd ed.). New York: McGraw-Hill. 
59. Dietrich, F. J., \& Long, R. K. (1969). An efficient moderate-size vertical-incidence ionosonde antenna for $2-20 \mathrm{MHz}$ polarization studies. IEEE Transactions on Antennas and Propagation, 17(5), 551-557.

60. Witte, A. (2008). A broadband antenna for ionospheric sounding. Stockholm: KtH MSc project.

61. Jones, T. O. (2014). Directional array for Near-Vertical-Incidence Skywave (NVIS). IEEE Transactions on Antennas and Propagation, 56(2), 132-143.

62. Barker, G. E., Taylor, J., \& Hagn, G. H. (1971). Summary of measurements and modeling of the radiation patterns of simple $\mathrm{HF}$ field antennas in open (Level) Terrain, Mountains, and Forests. Standford Research Inst., Menlo Park, CA, USA.

63. Hagn, G. H., \& Van der Laan, J. E. (1970). Measured antenna response of vertical whip antennas towards zenith using ionospheric sounder. EEE Transactions on Vehicular Technology, 19(3), 230-236.

64. Richie, J. E., \& Joda, T. (2003). HF antennas for NVIS applications mounted to helicopters with tandem main rotor blades. IEEE Transactions on Electromagnetic Compatibility, 45(2), 444-448.

65. Vlasic, R., \& Sumic, D. (2008). An optimized shipboard HF loop antenna for NVIS. In Presented at ELMAR, Zadar, Croatia.

66. Polycarpou, A. C., \& Balanis, C. A. (2000). Rotor modulation of helicopter antenna characteristics. In Presented at APS UT, Salt Lake City, USA.

67. Kraus, J. D. (1988). Antennas (2nd ed.). New York: McGraw-Hill.

68. ITU. (2013). Radio noise. ITU-R Rec. P.372-11, International Telecommunications Union, Geneva, Switzerland.

69. Coleman, C. J. (2002). A direction-sensitive model of atmospheric noise and its application to the analysis of HF receiving antennas. Radio Science, 37(3), 3.1-3.10.

70. Ellingson, S. (2005). Antennas for the next generation of low frequency radio telescopes. IEEE Transactions on Antennas and Propagation, 53(8), 2480-2489.

71. Warrington, E. M., Jackson, C. A., \& Lundborg, B. (2000). Directional diversity of $\mathrm{HF}$ signals received over high latitude paths, and the possibility of improved data throughput by means of spatial filtering. IEE Proceedings-Microwaves, Antennas and Propagation, 147(6), 487-494.

72. McNamara, L. F. (1991). The ionosphere: Communications surveillance and direction finding. Malabar: Krieger Publishing Company.

73. Breakall, J. K., Young, J. S., Hagn, G. H., Adler, R. W., Faust, D. L., \& Wemer, D. H. (1994). The modeling and measurement of $\mathrm{HF}$ antenna skywave radiation patterns in irregular terrain. IEEE Transactions on Antennas and Propagation, 42(7), 936-945.

74. Jenkins, R. W. (1995). Antenna amplitude and phase pattern measurements using an aircraft-towed transmitter, CRC Report 95-003. Ottawa: Communications Research Centre.

75. Krause, M. (2013). Calibration of the LOFAR antennas. Master Thesis, University of Nijmegen, The Netherlands.

76. Burgess, S. J., \& Evans, N. E. (1999). Short-haul communications using NVIS HF radio. Electronics and Communication Engineering Journal, 11(2), 95-104.

77. Tooby, M. H., Arthur, P. C., \& Cotterill, P. L. (1999). Investigations into the channel characteristics of HF NVIS links and the implications for high data rate transfer. Presented at IEE colloquium on frequency selection and management techniques for $\mathrm{HF}$ communications, London, UK.

78. Tooby, M. H., Arthur, P. C., Cotterill, P. L., Hoult, N. S., \& Whiffen, J. R. (2000). An assessment on the propagation characteristics of the NVIS channel. In Presented at HFRST (pp. 263-267).

79. ITU. (1992). Use of high frequency ionospheric simulators. ITU-R Rec. F.520-2, International Telecommunications Union, Geneva, Switzerland.
80. Vilella, C., Miralles, D., \& Pijoan, J. (2008). An antarctica-toSpain HF ionospheric radio link: Sounding results. Radio Science, 43(4), 1-17.

81. Ads, A., Bergadà, P., Vilella, C., Regué, J., Pijoan, J., \& Bardají, R. (2012). A comprehensive sounding of the ionospheric HF radio link from Antarctica to Spain. Radio Science, 48(1), 1-12.

82. Lowell Digisonde International. www.digisonde.com.

83. Digital Radio Mondiale. (2009). System specification. ETSI ES 201980 V3.1.1, European Telecommunications Standards Institute, Sophia-Antipolis, France.

84. Gil, U., Guerra, D., Pena, I., de la Vega, D., Angueira, P., \& M. V'elez and G. Prieto,. (2012). Analysis of the NVIS Channel Availability in the Medium Wave Band, presented at BMSB (pp. 1-6). Korea: Seoul.

85. Losada, I. (2009). DRM MW NVIS nighttime quality assessment and channel analysis. PhD Thesis, University of the Basque Country, Bilbao, Spain.

86. ITU. (1990). Ionospheric propagation and noise characteristics pertinent to terrestrial radiocommunication systems design and service planning. ITU-R Report P.266-7, International Telecommunications Union, Geneva, Switzerland.

87. McNicol, R. W. E. (1949). The fading of radio waves of medium and high frequencies. Proceedings of the IEE-Part III: Radio and Communication Engineering, 96(44), 517-524.

88. Brennan, D. G. (1961). Probability theory in communication system engineering. In E. Baghdady (Ed.), Lectures on communication system theory. New York: McGraw-Hill.

89. Essex, E. A. (1968). Periodic fading of ionospheric echoes. Journal of Atmospheric and Terrestrial Physics, 30(7), 1441-1446.

90. Nakagami, M. (1960). The m-distribution, a general formula of intensity of rapid fading statistical methods in radio wave propagation. Oxford: Pergamon Press.

91. Papoulis, A., \& Pillai, S. U. (2002). Probability, random variables and stochastic processes (4th ed.). New York: McGraw-Hill.

92. Proakis, J. G. (1995). Digital communications (3rd ed.). New York: McGraw-Hill.

93. ITU. (2007). Multipath propagation and parameterization of its characteristics. ITU-R Rec. P.1407-5, International Telecommunications Union, Geneva, Switzerland.

94. Cannon, P. S., Angling, M. J., Heaton, J. A. T., \& Shukla, A. K. (2005). The effects of space weather on radio systems. Effects of space weather on technology infrastructure (pp. 185-201). Berlin: Springer.

95. Sklar, B. (1997). Rayleigh fading channels in mobile digital communication systems part II: Mitigation. IEEE Communications Magazine, 35(9), 148-155.

96. Watterson, C. C., Juroshek, J. R., \& Bensema, W. D. (1970). Experimental confirmation of an HF channel model. IEEE Transactions on Communication Technology, 18(6), 792-803.

97. ITU. (2000). Testing of HF modems with bandwidths of up to about $12 \mathrm{kHz}$ using ionospheric channel simulators. ITU-R Rec. F.1487-0, International Telecommunications Union, Geneva, Switzerland.

98. Furman, W. N., \& Koski, E. (2009). Standarization of an intermediate duration HF channel variation model. In Presented at IRST (pp. 137-141). Edinburgh, UK.

99. Gil, U., Garcia, J. A., Garcia, J. L., Sanchez, J., Guerra, D., Pena, I., et al. (2013). Near vertical incidence skywave field strength time variability characterization in the medium wave band. Presented at EuCAP (pp. 2615-2618). Gothenburg, Sweden.

100. Guerra, D., Coleto, M. A., Melgar, L., Gil, U., Pena, I., Prieto, G., et al. (2013). Measurement based near vertical incidence skywave channel model in the medium wave band. In Presented at EuCAP (pp. 3403-3407). Gothenburg, Sweden. 
101. Hunsucker, R. D., \& Hargreaves, J. K. (2002). The high-latitude ionosphere and its effects on radio propagation. London, UK: Cambridge University Press.

102. Magdaleno, S., Altadill, D., Herraiz, M., Blanch, E., \& de la Morena, B. (2011). Ionospheric peak height behavior for low, middle and high latitudes: A potential empirical model for quiet conditions-Comparison with the IRI-2007 model. Journal of Atmospheric and Solar-Terrestrial Physics, 73(13), 1810-1817.

103. Warrington, E. M., Stoker, A. J., \& Siddle, D. R. (2006). Measurement and modeling of HF channel directional spread characteristics for northerly paths. Radio Science,41(2), 1-13.

104. Jodalen, V., Lundborg, B., \& Jacobsen, B. (2000). Channel characteristics of HF NVIS paths in Northern Scandinavia. In Presented at HFRST (pp. 269-273). Guildford, UK.

105. Cannon, P. S., Angling, M. J., \& Davies, N. G. (2000). DAMSON HF channel Characterisation-a review. Presented at MILCOM, $1,59-64$.

106. Lossmann, E., Meister, M.-A., \& Madar, U. (2011). On HF communication link parameter estimation in the Baltic Region. In Presented at APWC (pp. 812-814). Torino, Italy.

107. Jodalen, V., Bergsvik, T., Cannon, P., \& Arthur, P. C. (2001). Performance of HF modems on high-latitude paths using multiple frequencies. Radio Science, 36(6), 1687-1698.

108. Davies, K., Cotterill, P. L., Ponsionen, C., \& Jodalen, V. (2003). On-air test and evaluation of STANAG 4538. In Presented at IRST (pp. 13-18). Bath, UK.

109. NATO. (2000). Interoperability and performance standards for data modems, STANAG 4539. Brussels: NATO Standardization Organization.

110. Johnson, E. E. (2006). HF radio mesh networks. In Presented at MILCOM. Washington, DC, USA.

111. Johnson, E. E., Koski, E., Furman, W. N., Jorgenson, M., \& Nieto, J. (2012). Artech house: Third generation and wideband HF radio communications.

112. Furman, W. N., \& Nieto, J. W. (2012). Latest on-air testing of U.S. MIL-STD-188-110C Appendix D wideband HF data waveforms. In Presented at IRST, Edinburgh, UK, pp. 1-5.

113. US Department of Defense. (2011). Interoperability and performance standards for data modems, MIL-STD-188-110A. Department of Defense Standardization Program, Philadelphia, PA, USA: B and C.

114. Spezielle Communications Systeme GmbH \& Co. KG, Hanau, Germany. www.p4dragon.com/en/PACTOR-4.html.

115. ITU. (2013). Bandwidths, signal-to-noise ratios and fading allowances in HF fixed and land mobile radiocommunication systems. Rec. ITU-R F.339-8, International Telecommunications Union, Geneva, Switzerland.

116. Reynolds, A. B., \& Blair, W. D. (2001). Tactical high frequency communications in the land arena-The current state of the art, DSTO-CR-0214. Edinburgh, Australia: Defense Science and Technology Organization.

117. Antoniou, S., Christofi, L., Green, P. R., \& Gott, G. F. (2006). High rate data transmission in the mid-latitude NVIS HF channel. IEE Proceedings-Communications, 153(2), 272-278.

118. Christofi, L. (2001). Measurement of HF NVIS radio channel parameters with application to the design of very high rate modems. Ph.D. Thesis, University of Manchester, UK.

119. Lamy-Bergot, C., Herry, S., Bernier, J. Y., \& Hun, F. N. B. (2013). On-air tests results for HF XL wideband modem. Presented at Nordic HF, Faro, Sweden.

120. Beverage, H. H., \& Peterson, H. O. (1931). Diversity receiving system of RCA communications inc. for radiotelegraphy. Proceedings of the Institute of Radio Engineers, 19(4), 529-561.

121. Friis, H. T., \& Feldman, C. B. (1937). A multiple-unit steerable antenna for short-wave reception. Proceedings of the Institute of Radio Engineers, 25(7), 841-917.
122. Enserink, S., Kose, C., Fitz, M., Urie, M., \& McCourt, R. (2015). A model for dual polarized HF MIMO communications. In Presented at MILCOM, Tampa, USA.

123. Witvliet, B. A., Laanstra, G. J., van Maanen, E., \& Alsina-Pagès, R. M. (2016). A transportable hybrid antenna-transmitter system for the generation of elliptically polarized waves for NVIS propagation research. Davos: EuCAP.

124. Witvliet, B. A., van Maanen, E., Petersen, G. J., Westenberg, A. J., Bentum, M. J., Slump, C. H., et al. (2014). The importance of circular polarization for diversity reception and MIMO in NVIS propagation. The Hague: EuCAP.

125. Witvliet, B. A., van Maanen, E., Petersen, G. J., Westenberg, A. J., Bentum, M. J., Slump, C. H., et al. (2015). Characteristic wave diversity in near vertical incidence skywave propagation. Lisbon: EuCAP.

126. Ndao, P. M., Erhel, Y., Lemur, D., Oger, M., \& Le Masson, J. (2013). Development and test of a trans-horizon communication system based on a MIMO architecture. EURASIP Journal on Wireless Communications and Networking, 1, 1-13.

127. Ignatenko, M., Sanghai, S. A., Lasser, G., Allen, B., Smith, R., Notaros, M., \& Filipovic, D. S. (2016). Wide-band high-frequency antennas for military services. IEEE Antennas and Propagation Magazine, 58(6), 64-74.

128. Orga, F., Hervás, M., \& Alsina-Pagès, R. M. (2016). Flexible lowcost SDR platform for HF communications. IEEE Antennas and Propagation Magazine, 58(6), 49-56.

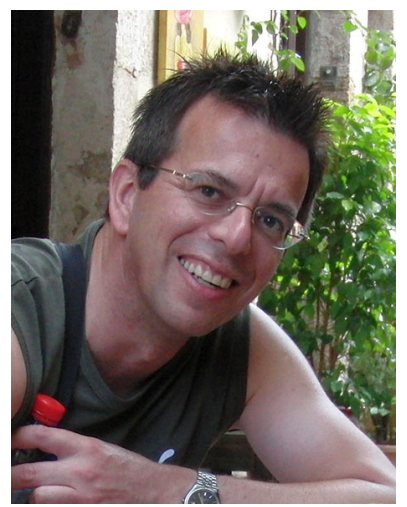

Ben A. Witvliet obtained his B.Sc. in Telecommunications from the Hogeschool voor Techniek en Gezondheidszorg, The Netherlands, in 1989. He worked as chief engineer and technical manager in high power broadcasting in Monaco, Madagascar and The Netherlands, and is currently employed by Radiocommunications Agency Netherlands as technical expert in spectrum management. In 2015 he obtained his Ph.D. in Telecommunication Engineering at the University of Twente, The Netherlands, and his NVIS research earned him the Anton Veder scientific radio research prize, which is issued yearly since 1929 . His main research interests are HF antennas, propagation and radio noise.

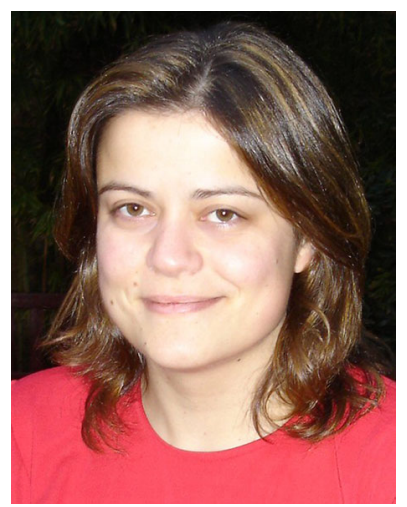

Rosa Ma Alsina-Pagès has a B.Sc. and M.Sc. in Electronics and Telecommunications in La Salle, Universitat Ramon Llull (URL) in 1999, 2001 and 2004 respectively. She obtained her Ph.D. in Telecommunication Engineering with Cum Laude in 2012, also in La Salle (URL). She coordinates the Signal Processing Research Line in the Research Group on Media Technologies. She has several papers in communications and signal processing journals. Her current research interests include digital signal processing and physical layer design. 